\title{
CAN WE REALLY PREVENT ALZHEIMER'S DISEASE?
}

\author{
Octavian-Mihai Sirbuㅁ, Anca-Maria Sandu', Florentina-Cristina Plesa², \\ Carmen-Adella Sirbu ${ }^{2}$ \\ ${ }^{1}$ University of Medicine and Pharmacy, Faculty of General Medicine, Bucharest \\ ${ }^{2}$ Department of Neurology, Central Universitary Emergency Military Hospital, \\ „Carol Davila“ University of Medicine and Pharmacy, Bucharest
}

\begin{abstract}
Alzheimer's disease, a neurodegenerative disorder that affects mainly the elderly people, was declared a public health priority by the World Health Organization. Because the main clinical manifestations are cognitive, behavioral and psychological symptoms, patients diagnosed with this disease have a significantly lower quality of life. This type of dementia has an inexorably progressive evolution so, families and health care staff, must make great efforts to provide a decent standard of living for these patients. Because it has a huge economic burden and there is no treatment to stop or cure the disease, it is important to emphasises modifiable risk factors and to apply prevention strategies, proved by scientific evidence.
\end{abstract}

Keywords: Alzheimer's, disease, dementia, prevention strategies

\section{INTRODUCTION}

Advances in the improvement of health services made in the last decade have led, among other things, to an increased life expectancy in the population. This has also contributed to a rise in the number of noncommunicable diseases related to age, including mental disorders. Dementia is a syndrome characterized by the disruption of several cognitive functions of the brain: learning capacity, language, judgment, memory, thinking, orientation, comprehension, calculation. Alzheimer's disease is the most common form, constituting $70 \%$ of the cases of dementia. Patients diagnosed with this disease have a significantly lower quality of life. Their families and health care staff must make great efforts to provide a decent standard of living for these patients.

In 2012, dementia was declared a public health priority by the World Health Organization. This highlights the fact that dementia is a common, severe dis- ease. Worldwide, there are more than 35 million people with dementia, among whom $25 \%$ are in Europe. Western Europe is the region with the highest number of people affected: 6,975,540 (World Health Organization). Global prevalence among the elderly was estimated at $10-13 \%$, and the rate increases exponentially with age: $47 \%$ of those aged over 85 years (1). It should be noted that, in general, many cases of dementia remain unidentified. In Romania there are around 35,000 patients with dementia, but the Romanian Society for Alzheimer estimates that more than $80 \%$ of the existing cases remain undiagnosed.

Due to low birth rates and increased life expectancy, the proportion of the working-age population is decreasing, while the number of people reaching retirement is rising (2). In addition, the number of age-dependent diseases, including Alzheimer's disease, will increase significantly. The number of people affected by it will double every 20 years to about 80 million by 2040 (3). 
The financial costs associated with the management of this disease are enormous, being a significant burden on the healthcare systems: the average cost of care for a patient with dementia is thrice the one for a patient without dementia (4). An estimate of future costs for patients with dementia in Europe predicts an increase of $43 \%$ compared to 2008 , reaching 250 billion in 2030 (5).

\section{CLINICAL SPECTRUM OF ALZHEIMER'S DISEASE}

Alzheimer's disease is a progressive degenerative disorder of the brain that occurs mostly in older people, causing more and more pronounced damage to cognitive functions of the brain, loss of the individual's intellectual abilities and social value, associated with behavioral disorders. Its incidence and prevalence increase exponentially with age. There are also inherited forms of the disease, which usually occur under the age of 65 , but they account for only $5 \%$ of the cases.

There is a wide range of clinical presentations; the onset is often slow and poorly perceptible. The major symptoms of the disease vary.

Memory Impairment. Is an emblematic early symptom of Alzheimer's disease. The memory pattern of memory loss is also specific. Declarative memory, which depends on the mesial and neocortical temporal structures, is the earliest and most deeply affected. Declarative memory, which stores the events that can be mentioned, discussed or declared, is subdivided into semantic memory and episodic memory. The latter is the most severely affected in the disease. In episodic memory, the memorized knowledge and events are attributed to a specific spatial temporal context. This side of memory gives us the feeling of time. At the same time, within episodic memory, we distinguish between immediate memory, the short and the long term memory. Out of these, the short-term memory is the first and most severely affected. (6) Semantic memory is represented by theoretical knowledge generally available, independent of time and place. This will be distorted in an advanced stage of the disease, being stored in the neocortex. Procedural memory, which stores the knowledge on how to perform or carry out certain actions or processes, remains intact until advanced stages.

Language. Changes in language fluency and anomia are among the earliest features of Alzheimer's disease. The first manifestations of language impairment occur as difficulty finding specific words (lapses), inability to periphrase, or the use of a limited vocabulary in everyday speech. In time, the patient gets to communicate in a poor vocabulary, with grammatical errors and even has difficulty comprehending.

Language dysfunction and impaired semantic memory are closely related. If a patient with Alzheimer's disease is asked to generate a list of words in a minute, they will score higher at the list of words that start with the same letter than the list of words belonging to a semantic field (7).

Visual spatial abilities. Patients often have difficulty in orientation in a new space, but also in familiar spaces in the advanced stages of the disease. Visual agnosia (inability to recognize objects) and prosopagnosia (inability to recognize people) occur late in the evolution of the disease.

Anosognosia. Most patients with Alzheimer's disease are often unaware of their slow performance or symptoms. They tend to find excuses or alibis for their deficiencies. There are patients with the ability to understand the disease, its symptoms and to admit it. They most frequently develop depression, which will adversely affect the disease.

On the other hand, patients who are not aware of their condition, become agitated, uninhibited or present psychotic disorders. (8)

Apraxia. Difficulty in planning and performing a series of movements to achieve a specific objective (in the absence of motor deficit) occurs in advanced stages of the disease. In most cases the degree of apraxia is decisive in assessing self-care ability. Patients cannot perform actions like dressing or eating.

Neuropsychiatric Disorders. With the progression of Alzheimer's disease, patients become agitated, anxious, and aggressive, provide evidence of personality changes, visual or auditory hallucinations.

\section{STRATEGIES OF PREVENTION}

\section{Risk factors}

To discuss prevention strategies, it is imperative to first know the risk factors, especially as the causes of the disease are uncertain. A number of risk factors genetic, constitutional or acquired, environmental factors - have been incriminated. Most links between the alleged risk factors and the disease have been made on the basis of numerous observational studies, but randomized trials are essential to ascertain whether there is a causal relationship between a particular factor and the disease. An estimated one-third of the cases are attributed to modifiable risk factors (9). Consequently, we will present those modifiable risk factors whose management at an early age may decrease the incidence of Alzheimer's disease in the population. 
1. Cerebrovascular disease. In more than $50 \%$ of the cases, Alzheimer's disease and cerebrovascular disease coexist. Given the lax pathological definition of vascular dementia and Alzheimer's disease, the patients with clinical manifestation of dementia display a rather intricate pathological mechanism. This association is known as mixed dementia (10). Clinical pathological studies have shown that cerebrovascular diseases lower the age of clinical manifestation of dementia within the Alzheimer's disease. So patients who also have cerebrovascular disorders will develop earlier and more severe cognitive impairment (11).

2. Hypertension. Is a known cardiovascular risk factor, but when it comes to its association with the risk of dementia things are not so clear. Although numerous longitudinal studies have observed that high blood pressure correlates with a higher incidence of dementia, this link does not seem to be a direct one. Thus, in a study on 9,704 female patients, hypertension was correlated with decreased cognitive performance. Nevertheless, at the same time, most of those with poor results had also suffered a stroke (12). Basically, this study did not show a direct association between hypertension and decreased cognitive function, but rather between hypertension and cerebrovascular disease (stroke), which in turn led to lower cognitive abilities. Yet the same study reveals that patients suffering from hypertension before the stroke showed a more pronounced decrease in cognitive function than those who had the stroke without prior hypertension. Thickening and stiffening of blood vessels and their loss of elasticity due to high blood pressure have been linked to the development of various types of dementia and the progression of beta amyloid plaque deposits (13).

3. Head injuries. Traumatic brain injuries are among the oldest conditions described in human history and remain a major source of morbidity and mortality. Dementia represents one of their most dreaded complications in the long term. The data show that moderate trauma leads to an increased risk of dementia by 2 to 4 times. It is well-known that among certain categories of people (boxers, hockey and rugby players, military staff) there is a high risk of developing a distinct type of pathology: Chronic traumatic encephalopathy (CTE) (14). However, the pathophysiological similarities between this and Alzheimer's disease are still unknown.

4. The use of benzodiazepines. It was noted that patients who are under medication with benzodiazepines develop specific cognitive disorders in time. Thus, the use of benzodiazepines for a period exceed- ing 180 days was associated with an increased risk of developing Alzheimer's disease by 1.5 times (15). The majority of adults diagnosed with dementia had a personal history of an average of 4-5 years of benzodiazepine use. This long term association suggests a direct effect on the disease pathogenesis. However, benzodiazepine medication is likely to have been prescribed for prodromal Alzheimer's disease, for a condition associated with an increased risk of dementia. Although there is no consensus in this regard, prescribing these drugs should be well justified and this possible long-term adverse effect should be considered when weighing the risks versus the benefits.

5. Diabetes Mellitus. The relationship between diabetes and major types of dementia is a matter of controversy. There are numerous studies associating diabetes with an increased risk of cognitive impairment and dementia. This risk may increase with $50-100 \%$ for Alzheimer's disease and up to $150 \%$ for vascular dementia (16). There is also an association between diabetes and cerebrovascular diseases, which in turn are correlated with an increased risk of dementia. At the same time, we have evidence linking the elevated levels of glucose to atrophy in the hippocampus and amygdala, frequently correlated to Alzheimer's disease (17). Other studies have shown the involvement of insulin degrading enzyme, with a role in the metabolism of amyloid beta, in the accumulation of beta-amyloid plaques in the CNS (18).

6. Obesity and increased body mass index (BMI). Various studies have analyzed the correlation between weight and the risk of developing Alzheimer's disease. It has been noted that overweight or obese middle aged people will have an increased risk of developing dementia, obesity inducing accelerated aging at the cerebrovascular level (19). At the same time, an increase in BMI was correlated with a decrease in cognitive performance in persons without dementia (20). On the other hand, numerous studies have shown the association of weight loss or decrease in BMI in old age with an increased risk of Alzheimer's disease. This may be due to the fact that weight loss is an early symptom of the disease rather than a risk factor in itself.

7. Smoking. Several prospective studies have suggested that smoking among the middle aged and the elderly is associated with an increased risk of dementia. A meta-analysis concluded that smokers have an increased risk of developing Alzheimer's disease, vascular dementia or any other type of dementia by 1.3 to 1.7 times. They also get poor results in the Mini-Mental State examination. (21) Exposure to cigarette smoke is also correlated with an increased risk of developing Alzheimer's disease. (22) 
8. Pesticides. Several studies have shown that occupational exposure to pesticides may increase the risk of developing Alzheimer's disease. The commonly used organochlorine and organophosphorus pesticides inhibit acetylcholinesterase in the central nervous system synapses and can produce long-term adverse effects.

Given that Alzheimer's disease is a progressive, disabling condition, with a major negative impact on not only the patient but also the family and health systems, and that there is no cure or stopping of the progression of the disease, the winning solution seems to be focusing on prevention and early detection.

\section{PREVENTION STRATEGIES}

\section{Intellectual activity}

Primary prophylaxis may slow down or delay the development of the disease. Scientists have shown that, among other risk factors, the lack of intellectual activity is the one most commonly associated to dementia. People who are more educated and involved in highly complex mental activities have lower rates of Alzheimer's disease (23). A high level of education is considered to be a greater cognitive reserve that decreases the impact of the disease (24). Neural plasticity (perhaps through synaptic reorganization) seems to underlie the protective mechanism by which high education levels delay the clinical manifestation of the disease. Even if a person had no access to higher education in their youth, intellectual activities in mature or old age can be just as useful. Studies show that older adults with intense, regular cognitive activity run a lower risk of developing dementia, regardless of their previous training (25). Therefore, reading books, learning a new foreign language, doing puzzles or taking part in memory and logic games, or learning to dance, can be an effective way to delay or even prevent Alzheimer's disease.

\section{Socialization}

Quite often, older people may come to live in solitude. Due to retirement, people who used to be active become isolated, and besides mental inactivity there will be estrangement and alienation from former co-workers or friends. They may also separate from family. The death of the spouse can contribute fundamentally to this scenario. In these circumstances the risk of developing depression, cognitive impairment, and dementia is significant. Maintaining social interaction with former colleagues, friends, family mem- bers or membership to new circles of people with common interests (hobbies) is a key element in preserving cognitive integrity and having a healthy aging. Social networking, setting goals, preserving intellectual and socio-economic preoccupations, involvement in challenging cognitive activities and maintaining social relationships allegedly have a protective role against Alzheimer's disease. (26)

\section{Physical activity}

Exercise reduces the risk of cognitive impairment and dementia through both the direct neurotrophic effect of physical activity and the improvement of the cardiovascular and cerebrovascular risk profiles. Moderate physical activity can decrease the risk of cognitive decline to $37 \%$ (27).

\section{Diet}

Several cohort studies have examined a possible relationship between dietary components and the risk of Alzheimer's disease and other forms of dementia. A higher rate of cognitive decline or dementia incidence was observed in people with increased dietary intake of saturated fat and cholesterol (28). However, cholesterol-lowering therapies such as statins have a questionable effect on dementia. The possible beneficial effect of a diet of fish, rich in omega 3 fatty acids, on dementia and decreased cognitive performance has been the subject of several studies. Some of them have shown the benefit of fish consumption (29). But there is no consensus in this regard. A diet rich in fruits and vegetables is supported by the benefits brought about by lowering the cardiovascular risk. In a study on a population of 8,085 people, the daily consumption of fruits and vegetables was associated with a decreased risk of dementia (29). The Mediterranean diet consisting of fruits and vegetables, whole grains, beans, nuts and seeds, olive oil, with a moderate content of fish products and poultry, and a low content of red meat has been associated with a decrease in the incidence of Alzheimer's disease (30). The consumption of flavonoid-rich berries (blueberries, strawberries) has been associated with lower rates of cognitive decline. It is assumed that flavonoids have an antioxidant and anti-inflammatory beneficial role (31).

\section{CONCLUSION}

To conclude, Alzheimer's disease is a neurodegenerative disorder of unknown cause and pathogenesis, which primarily affects the elderly. The main clinical 
manifestations are selective memory impairment and dementia. Alzheimer's disease is inexorably progressive, but the pace of progression may vary. The average life expectancy was reported to be between 3 and 8 years (32). There are treatments that can modulate the course of the disease and / or relieve some symptoms, but there is no treatment to stop or cure the disease. Under these circumstances, to analyze and try to avoid the risk factors on the one hand, and to promote and apply the protective factors in order to prevent or delay the onset of the disease on the other hand, seems to be the ideal strategy to fight this disease.

\section{REFERENCES}

1. Jee-Young Han, Seol-Heui Han. Primary Prevention of Alzheimer's Disease: Is It an Attainable Goal? J Korean Med Sci. 2014 Jul; 29(7):886-892. English.

2. Eurostat: http://epp.eurostat.ec.europa.eu/statistics_explained/index. php/Population_structure_and_ageing

3. Cleusa P. Ferri et al. Global prevalence of dementia: a Delphi consensus study. The Lancet, Volume 366, Issue 9503, Pages 2112 - 2117, 17 December 2005

4. T.S. Dharmarajan, Srinivas G. Gunturu. Alzheimer's Disease: A Healthcare Burden of Epidemic Proportion. Am Health Drug Benefits. Jan 2009; 2(1): 39-47.

5. Anders Wimo, Linus Jönsson, Anders Gustavsson. Cost of illness and burden of dementia in Europe - Prognosis to 2030. http://alzheimer.vo.lu/

6. Peters F., Collette F., Degueldre C., Sterpenich V., Majerus S., Salmon E. The neural correlates of verbal short-term memory in Alzheimer's disease: an fMRI study. Brain. 2009; 132(Pt 7):1833.

7. Marczinski C.A., Kertesz A. Category and letter fluency in semantic dementia, primary progressive aphasia, and Alzheimer's disease. Brain Lang. 2006; 97(3):258

8. Harwood D.G., Sultzer D.L., Wheatley M.V. Impaired insight in Alzheimer disease: association with cognitive deficits, psychiatric symptoms, and behavioral disturbances. Neuropsychiatry Neuropsychol Behav Neurol. 2000; 13(2):83.

9. Norton S., Matthews F.E., Barnes D.E., Yaffe K., Brayne C. Potential for primary prevention of Alzheimer's disease: an analysis of population-based data. Lancet Neurol. 2014 Aug;13(8):788-94

10. Neuropathology Group. Medical Research Council Cognitive Function and Aging Study. Pathological correlates of late-onset dementia in a multicentre, community-based population in England and Wales. Neuropathology Group of the Medical Research Council Cognitive Function and Ageing Study (MRC CFAS). Lancet. 2001; 357(9251):169.

11. Riekse R.G., Leverenz J.B., McCormick W., Bowen J.D., Teri L., Nochlin D., Simpson K., Eugenio C., Larson E.B., Tsuang D. Effect of vascular lesions on cognition in Alzheimer's disease: a community-based study. J Am Geriatr Soc. 2004;52(9):1442

12. Elkins J.S., Yaffe K., Cauley J.A., Fink H.A., Hillier T.A., Johnston S.C. Pre-existing hypertension and the impact of stroke on cognitive function. Ann Neurol. 2005; 58(1):68

13. Hughes T.M., Kuller L.H., Barinas-Mitchell E.J., McDade E.M., Klunk W.E., Cohen A.D., Mathis C.A., Dekosky S.T., Price J.C., Lopez O.L. Arterial stiffness and $\beta$-amyloid progression in nondemented elderly adults. JAMA Neurol. 2014; 71(5):562

14. Moretti L., Cristofori I., Weaver S.M., Chau A., Portelli J.N., Grafman J. Cognitive decline in older adults with a history of traumatic brain injury. Lancet Neurol. 2012 Dec; 11(12):1103-12
15. Billioti de Gage S., Moride Y., Ducruet T., Kurth T., Verdoux H., Tournier M., Pariente A., Bégaud B. Benzodiazepine use and risk of Alzheimer's disease: case-control study. BMJ. 2014

16. Biessels G.J., Staekenborg S., Brunner E., Brayne C., Scheltens P. Risk of dementia in diabetes mellitus: a systematic review. Lancet Neurol. 2006; 5(1):64

17. Cherbuin N., Sachdev P., Anstey K.J. Higher normal fasting plasma glucose is associated with hippocampal atrophy: The PATH Study. Neurology. 2012; 79(10):1019.

18. Farris W., Mansourian S., Chang Y., Lindsley L., Eckman E.A., Frosch M.P., Eckman C.B., Tanzi R.E., Selkoe D.J., Guenette S. Insulin-degrading enzyme regulates the levels of insulin, amyloid beta-protein, and the beta-amyloid precursor protein intracellular domain in vivo. Proc Natl Acad Sci USA. 2003 Apr; 100(7):4162-7.

19. Whitmer R.A., Gunderson E.P., Barrett-Connor E., Quesenberry C.P. Jr., Yaffe K. Obesity in middle age and future risk of dementia: a 27 year longitudinal population based study. BMJ. 2005; 330(7504):1360

20. Cournot M., Marquié J.C., Ansiau D., Martinaud C., Fonds H., Ferrières J., Ruidavets J.B. Relation between body mass index and cognitive function in healthy middle-aged men and women. Neurology. 2006; 67(7):1208

21. Anstey K.J., von Sanden C., Salim A., O'Kearney R. Smoking as a risk factor for dementia and cognitive decline: a meta-analysis of prospective studies. Am J Epidemiol. 2007; 166(4):367

22. Chen R. Association of environmental tobacco smoke with dementia and Alzheimer's disease among never smokers. Alzheimers Dement. 2012 Nov; 8(6):590-5.

23. Prince M., Acosta D., Ferri C.P., Guerra M., Huang Y., Llibre Rodriguez J.J., Salas A., Sosa A.L., Williams J.D., Dewey M.E., Acosta I., Jotheeswaran A.T., Liu Z. Dementia incidence and mortality in middle-income countries, and associations with indicators of cognitive reserve: a 10/66 Dementia Research Group population-based cohort study. Lancet. 2012 Jul; 380(9836):50-8. Epub 2012 May 23.

24. Ngandu T., von Strauss E., Helkala E.L., Winblad B., Nissinen A., Tuomilehto J., Soininen H., Kivipelto M. Education and dementia: what lies behind the association? Neurology. 2007; 69(14):1442

25. Vemuri P., Lesnick T.G., Przybelski S.A., Machulda M., Knopman D.S., Mielke M.M., Roberts R.O., Geda Y.E., Rocca W.A., Petersen R.C., Jack C.R. Jr. Association of lifetime intellectual enrichment with cognitive decline in the older population. JAMA Neurol. 2014 Aug; 71(8):1017-24.

26. P.A. Boyle, A.S. Buchman, R.S. Wilson, L. Yu, J.A. Schneider, D.A. Bennett. Effect of purpose in life on the relation between Alzheimer disease pathologic changes on cognitive function in advanced age. Arch Gen Psychiatry, 69 (5) (2012), pp. 499-505 
27. Sofi F., Valecchi D., Bacci D., Abbate R., Gensini G.F., Casini A., Macchi C. Physical activity and risk of cognitive decline: a metaanalysis of prospective studies. J Intern Med. 2011 Jan; 269(1):107-17.

28. Morris M.C., Evans D.A., Bienias J.L., Tangney C.C., Wilson R.S. Dietary fat intake and 6-year cognitive change in an older biracial community population. Neurology. 2004; 62(9):1573

29. Barberger-Gateau P., Raffaitin C., Letenneur L., Berr C., Tzourio C., Dartigues J.F., Alpérovitch A. Dietary patterns and risk of dementia: the Three-City cohort study. Neurology. 2007; 69(20):1921
30. Scarmeas N., Luchsinger J.A., Mayeux R., Stern Y. Mediterranean diet and Alzheimer disease mortality. Neurology. 2007; 69(11):1084

31. Devore E.E., Kang J.H., Breteler M.M., Grodstein F. Dietary intakes of berries and flavonoids in relation to cognitive decline. Ann Neurol. 2012 Jul; 72(1):135-43.

32. Adak S., Illouz K., Gorman W., Tandon R., Zimmerman E.A., Guariglia R., Moore M.M., Kaye J.A. Predicting the rate of cognitive decline in aging and early Alzheimer disease. Neurology. 2004;63(1):108 